\title{
ANTI-INFLAMMATORY AND ANTI-PLATELET ACTIVITIES OF AVENA SATIVA ARE MEDIATED THROUGH THE INHIBITION OF CYCLOOXYGENASE AND LIPOXYGENASE ENZYMES
}

\author{
Sagheer Ahmed ${ }^{1,5}$, Saima Gul ${ }^{2}$, Humera Gul ${ }^{3} \&$ Muhammad Hanif Bangash $^{4}$ \\ PAPRSB Institute of Health Sciences, Universiti Brunei Darussalam \\ Department of Zoology, Abdul Wali Khan University, Mardan, Pakistan \\ Department of Botany, Abdul Wali Khan University, Ma rdan, Pakistan \\ Chashma Nuclear Power Plant, Chashma city, Pakistan \\ Department of Pharmacy, Kohat University of Science \& Technology, Kohat, Pakistan
}

Corresponding author: sagheer.ahmed@ubd.edu.bn

\begin{abstract}
Avena sativa (AS) is a well-known food crop and an important medicinal plant. It has been used for the treatment of various diseases, particularly for the treatment of inflammatory and cardiovascular diseases. However, the scientific rationale and mechanisms by which it functions in these diseases is still un- known. This study was designed to explore the inhibitory activity of AS aqueous, n-hexane and butanolic fractions on arachidonic acid (AA) metabolism. For this purpose these fractions of AS were screened for the presence of activities against AA metabolites and their effectiveness was further evaluated by studying platelet aggregation induced by AA, adenosine diphosphate (ADP), platelet activating factor (PAF), and collageAA metabolism was studied by thin layer chromatography system while platelet aggregation was measured by dual channel Lumiaggregometer.Aqueous and n-hexane fractions of AS were totally ineffective against AA metabolism and platelet aggregation. However, butanolic fraction inhibited the AA metabolites- thromboxane B2 (TXB2) through cyclooxygenase (COX) pathway and lipoxygenase product-1 (LP-1) and 12-hydroxyeicosatetraenoic acids (12-HETE) through lipoxygenase (LOX) pathway. Similarly butanolic fraction of AS showed strong inhibition against AA, PAF-induced aggregation but was less potent against ADP.AS possesses components which can inhibit AA metabolism and platelet aggregation. This may be one of the underlying mechanisms of their actions in cardiovascular and inflammatory diseases.
\end{abstract}

\section{KEY WORDS}

AS, bronchodilator, cyclooxygenase, lipoxygenase, aqueous fraction, butanolic fraction.

\section{INTRODUCTION}

AS (oat) is an annual crop used for nutrition by human and animal and even before being used as a food, it was used as a medicinal plant (Butt, 2008). Therefore, AS has a unique profile as a cereal because of its nutritional value as a whole grain cereal and as an important medicinal plant (Masood, 2008). Traditional medicinal uses of AS include improving cognitive performance and mental health (Wichtl, 2002). AS reduces both low-density lipoprotein (LDL) as well as total cholesterol (Truswell, 2002). Oat extract was also found to inhibit a number of pro-inflammatory cytokines and adhesion molecules (Liu, 2004). AS reduces blood pressure (Saltzman, 2001) and used to treat stress, tension, excitation and anxiety (Schellekens, 2009). AS is also reported to improve endothelial function (Katz, 2001), and indicated in cardiovascular diseases as well as some metabolic diseases such as diabetes (Feng, 2013). AS contains a number of different compounds including phytic acid, vitamin E, soluble fibers, avenanthramides (Avns)- a type of phenoles (Dimberg, 2005), as well as astocotrienols, flavonoids, phenolic acids and sterols (Peterson, 2001). Avn-c is not only the most abundant of the three Avns, a, b and $c$ (Matsukawa et al 2000) but also possesses the most potent antioxidant activity (Ji, 2003). Nie (2006a) reported that proliferation of vascular smooth muscle cells is inhibited by Avnsc. Avns-c also enhanced the production of nitric oxide (NO) and increased expression of endothelial NO synthase (Nie, 2006b). AS is used traditionally in cardiovascular and ischemic diseases, however, the scientific principle of its use is not known. Studies by various investigators suggest that inhibition of platelet aggregation is a common feature of a number of medicinal plant used traditionally in cardiovascular diseases (Gul, 2010, Zia-Ul-Haq, 2012a,b, Hussain, , 2010, Imran, 2102). Therefore, in the present investigation, studies are carried out to screen AS against human platelet aggregation induced by diversity of agonists and on AA metabolism through COX and LOX pathways.

\section{MATERIAL AND METHODS}

All organic solvents, chemicals and reagents used in the experiments were of analytical and highest purity grade. All the chemicals are purchased from Sigma chemical company, St. Louis, USA except 14C arachidonic acid which was purchased from Amersham Biosciences; acetic acid and citric acid from BDH and sodium phosphate (mono and dibasic) was purchased from Merck, USA.

\section{EXTRACTION OF PLANT MATERIAL}

Four Kiligrams of AS grains were purchase from Kohat District, Khyber Pakhtunkhwa, Pakistan. The grains of AS were ground using a commercial grinder to obtain fine powder. Fifteen hundred (1500) grams of ground material was soaked in 5 liter aqueous methanol and left for 24 hours at $4{ }^{\circ} \mathrm{C}$. The extract was filtered using a filtration flask and whattmann paper. This extract was evaporated on a rotary evaporator under reduced pressure (- 760 $\mathrm{mmHg}$ ) to a thick, semi-solid mass of dark brown color i.e. crude extract.

\section{FRACTIONATION OF THE CRUDE EXTRACT}

Five hundred (500) grams of the extract was dissolved in distilled water. This was then introduced in a separating funnel. n-hexane (50-70 ml) was then added into the same separating funnel. This mixture was shaken vigorously, regularly allowing the air to escape out. It was kept for about $30 \mathrm{~min}$ at room temperature to let the two layers separate. The upper layer of $n$-hexane was acquired and the same procedure was repeated twice and all the n-hexane layers were collected and concentrated in a rotary evaporator to obtain the $\mathrm{n}$-hexane fraction. Butanol $(50 \mathrm{ml})$ was then added to the remaining layer and the same process was repeated as with n-hexane, finally obtaining the butanol fraction, the yield of both fractions was $22 \%$ and $15 \%$, respectively, while the remaining layer was filtered using a filtration flask and whattmann paper and used in the experiments as aqueous fraction.

\section{ARACHIDONIC ACID METABOLISM BY HUMAN} PLATELETS

Archidonic acid metabolism and thromboxane B2 (TXB2) formation was studied using a Berthold TLC linear analyzer and 
chromatography data system (Model LKB 511, Berthold, W. Germany) as described previously (Saeed, 2007a). Human blood platelets were obtained in plastic bags containing $30-40 \mathrm{ml}$ concentrated platelet rich plasma (PRP) from the diagnostic laboratory of the Aga Khan University Hospital, Karachi. The PRP was centrifuged at $1200 \mathrm{~g}$ for $20 \mathrm{~min}$ and the sedimented platelets were washed twice with an ice-cold phosphate buffer $(50 \mathrm{mM}, \mathrm{PH}$ 7.4), containing $\mathrm{NaCl}(0.15 \mathrm{M})$ and EDTA $(0.2 \mathrm{mM})$. After centrifugation platelets were re-suspended in the same buffer without EDTA at the initial PRP concentration. The PRP suspension was homogenized at $4{ }^{\circ} \mathrm{C}$ using a polytron homogenizer for $15 \mathrm{sec}$ and the homogenate centrifuged at $1200 \mathrm{~g}$ for $20 \mathrm{~min}$. Supernatant $(300 \mu \mathrm{l}$ containing $0.4 \mathrm{mg}$ protein) was incubated with $10 \mu \mathrm{g}$ unlabelled $\mathrm{AA}$ and $0.1 \mu \mathrm{Ci}[1-14 \mathrm{C}] \mathrm{AA}$ in the presence and absence of test fraction. After $15 \mathrm{~min}$ of gentle shaking in air at 37 ${ }^{\circ} \mathrm{C}$ the reaction was stopped by adding $0.4 \mathrm{ml}$ citric acid $(0.4 \mathrm{M})$ and ethyl acetate $(7.0 \mathrm{ml})$. After mixing and centrifuging at $600 \mathrm{~g}$ for 5 $\min$ at $4{ }^{\circ} \mathrm{C}$, the organic layer was separated and evaporated to dryness under nitrogen. Residues were dissolved in $40 \mu 1$ of ethanol and $20 \mu \mathrm{l}$ was applied to silica gel $\mathrm{G}$ thin layer chromatography (TLC) plates (Analtech Delaware, USA). The AA, TXB2 (a stable degradation product of TXA2), LP1 and 12-HETE standards were plotted separately.

\section{SOLVENT SYSTEMS FOR DEVELOPING TLC PLATES}

The TLC plates were developed in ether/petroleum ether [boiling range 40-60]/acetic acid (50:50:1, v/v) to a distance of $17 \mathrm{~cm}$. By use of this solvent system the various lipoxygenasse products such as 12-hydroxy-eicosatetraenoic acid (HETE) were separated with TXB2 and prostaglandins remaining at the origin. The solvent system used for the separation of TXB2 was ethyl acetate/isooctane/water/acetic acid (11:5:10:2, v/v upper phase). Radioactive zones were located and quantified by use of a Berthold TLC linear analyzer and chromatography data system (Model LKB 511, Berthold, Germany).

\section{PREPARATION OF PLATELETS}

Platelet effects were studied in human platelets by taking blood via venipuncture from normal human volunteers reported to be free of medication for 7 days. Blood sample were mixed with $3.8 \%(\mathrm{w} / \mathrm{v})$ sodium citrate solution (9:1) and centrifuged at $260 \times \mathrm{g}$ for $15 \mathrm{~min}$ at $20{ }^{\circ} \mathrm{C}$ to obtain platelet-rich plasma (PRP). The remaining blood samples were centrifuged at $1200 \times \mathrm{g}$ for $10 \mathrm{~min}$ to obtain plateletpoor plasma (PPP). Platelet count was determined by phase contrast microscopy and all aggregation studies were carried out at $37^{\circ} \mathrm{C}$ with PRP having platelet counts between 2.5 and $3.0 \times 108 \mathrm{ml}-1$ of plasma (Aslam, 2008).

\section{MEASUREMENT OF PLATELET AGGREGATION}

Aggregation was monitored using dual-channel Lumiaggregometer (Model 400 Chronolog Corporation, Chicago, USA) using $0.45 \mathrm{ml}$ aliquots of PRP (Saeed, 2006, Gul, 2010). The final volume was made up to $0.5 \mathrm{ml}$ with the test fraction. Aggregation was induced by AA $(1.7 \mathrm{mM}), \operatorname{ADP}(2.2 \mu \mathrm{M}), \operatorname{PAF}(0.8 \mu \mathrm{M})$ and collagen. The antiplatelet effects of test fractions were studied by pretreatment of PRP with each fraction for $2 \mathrm{~min}$ followed by addition of platelet agonist. The resulting aggregation was recorded for $4 \mathrm{~min}$ after challenge by the change in light transmission as a function of time. After establishing the anti-platelet activity against various agonists, dose-response curves were constructed to calculate the $1 \mathrm{C} 50$ values.

\section{STATISTICAL ANALYSIS}

Statistical analysis was done by student's t-test. $\mathrm{P}<0.05$ was considered significant. All the experiments were performed in triplicate.

\section{RESULTS}

No activity was found in the n-hexane and aqueous fractions. The only active fraction was butanolic fraction. The butanolic fraction inhibited human platelet aggregation in a range of $1-10 \mathrm{mg}$, in a dose dependent manner. Platelet aggregation induced by AA, ADP and PAF was inhibited significantly by the butanolic fraction compared to the negative control group. The mean IC50 values in $\mathrm{mg}($ mean \pm SEM) against different aggregating agents were AA: $1.13 \pm 0.16$, ADP: $3.81 \pm 0.42$, PAF: $1.96 \pm 0.28$, (figure $1 B, C$ and D) while only $20 \%$ inhibition was observed against collagen-induced aggregation up to a dose of $10 \mathrm{mg}$ (figure 1A).

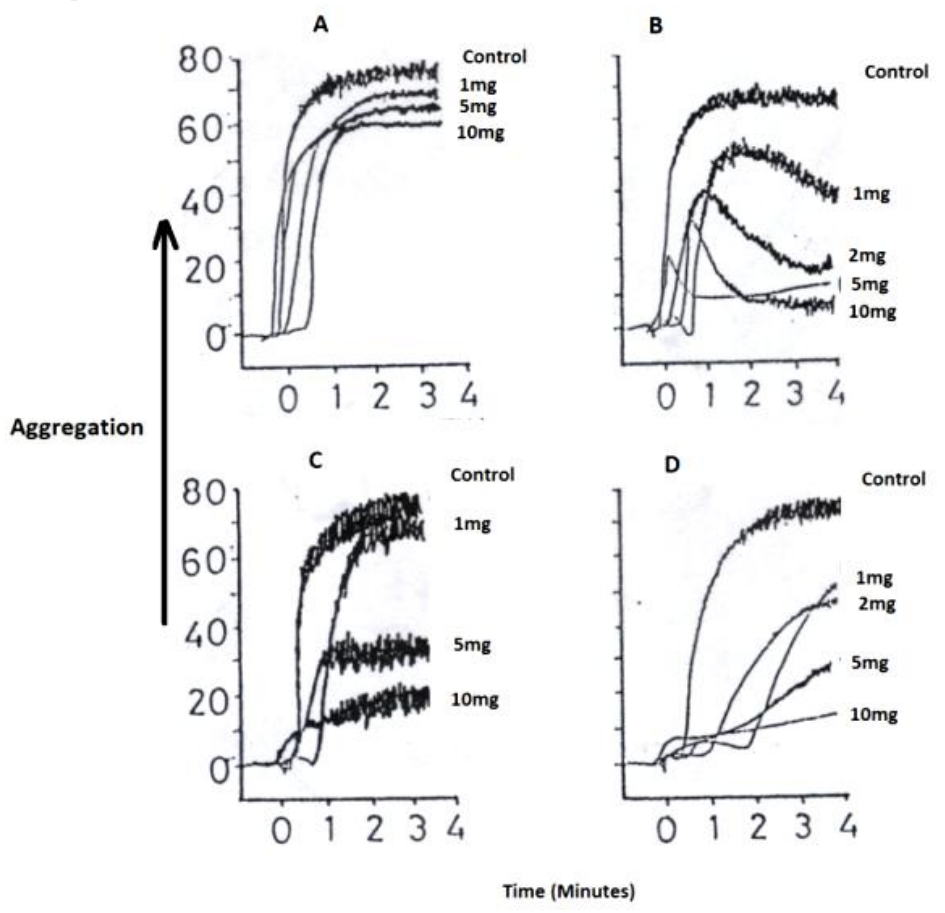

Figure 1: A representative scan of the pharmacological actions of the butanolic fraction of AS, showing inhibition of human platelet aggregation induced by collagen (A), AA (B), ADP (C), and PAF (D). As seen in the figure, butanolic fraction of AS was most potent against AA-induced aggregation $(B)$ and least potent against collagen-induced aggregation (A), $\mathbf{n = 4 - 6 .}$ 
The negative control had less than $5 \%$ effect at $50 \mu 1$, while positive control (Asprin) showed complete inhibition at $1 \mathrm{mg}$. These results showed that butanolic fraction inhibited AA-induced aggregation preferentially than that induced by any other agent. The degree of potency against different aggregating agents in decreasing order was $\mathrm{AA}>\mathrm{PAF}>\mathrm{ADP}$. The mean IC50 values in $\mathrm{mg}$ (mean $\pm \mathrm{SEM})$ of

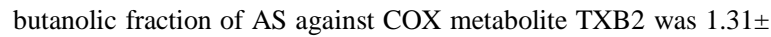
0.21, against LOX metabolites 12-HETE (2.56 \pm 0.45$)$ and LP1 $(1.84 \pm 0.31)$. These results showed that butanolic fraction inhibited AA metabolism through COX preferentially than through LOX (figure 2). The degree of potency against different metabolites in decreasing order was TXB2>LP-1>12-HETE.
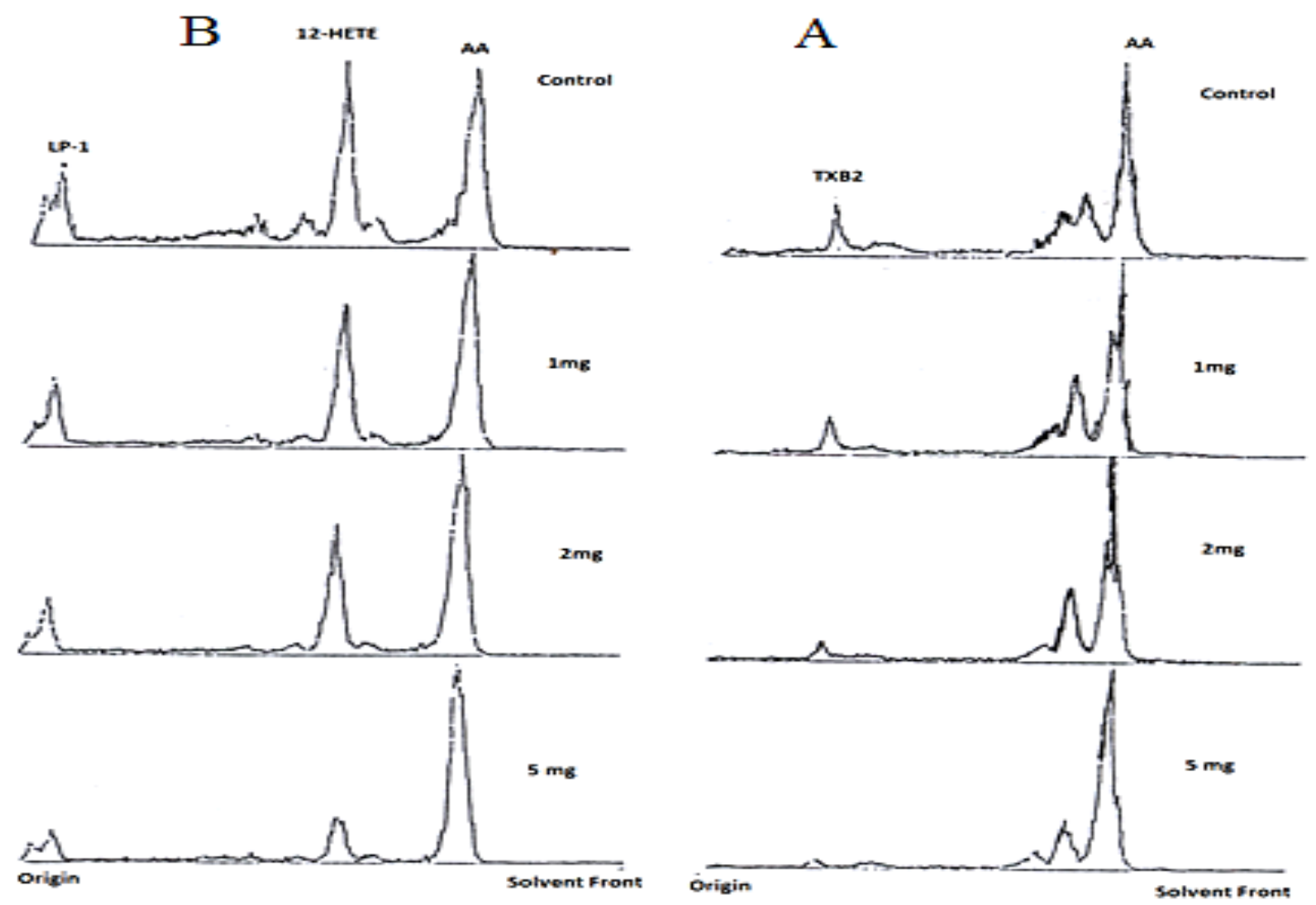

Figure 2: A scan showing butanolic fraction of AS inhibits AA metabolism through COX as well as LOX pathways. Complete inhibition of COX metabolite (figure 2A) at a lower dose suggests AS is more potent in blocking COX pathway than LOX pathway (figure 2B), $n=5$.

\section{DISCUSSION}

The search for new compounds to control the AA metabolites is a promising area of research (Saeed, 2003a). Natural compounds produced by the secondary metabolism of plants are potentially important sources of dual inhibition of COX and LOX pathways of AA metabolism (Saeed, 2004). The knowledge about natural inhibitors of AA pathway is scarce and practically non-existent for the control of various inflammatory conditions (Ahmed, 2013). In this study we screened n-hexane, aqueous and butanolic fractions of AS for the presence of inhibitory constituents against AA metabolism and platelet aggregation.Butanolic fraction of AS showed most potent activity against AA-induced platelet aggregation, suggesting it probably interferes with the COXmediated production of AA metabolites. This was further supported by the inhibition of TXB2 (a stable analogue of TXA2) by the butanolic fraction. TXA2 is one of the most potent aggregating agents in the body, and its inhibition by the butanolic fraction of AS points to the mechanism behind its traditional use in cardiovascular diseases. Similar activities for other plants have been reported by other investigators (Zia-Ul-Haq, 2012c, Ahmad, 2011a, Hussain, 2009). Butanolic fraction of AS also inhibited both LOX metabolites LP-1 and 12-HETE. However, it was more potent against LP-1 than against 12-HETE. Inhibition of COX and LOX pathways of AA metabolism is not unique to AS. A number of plants traditionally used in inflammatory and cardiovascular diseases are known to have the ability to inhibit enzymes responsible for AA metabolism (Bukhari, 2010, Ahmad, 2011b). Apart from plants, human plasma and lipoproteins are also reported to possess significant inhibitory activities against AA metabolites and platelet aggregation and form an important endogenous protective system (Saeed, 2007b, Aslam, 2008). Our study suggests that AA metabolism inhibitory constituents as well as platelet inhibitory constituents of AS are concentrated in the butanolic fraction. Therefore, it is likely that traditionally reported antiinflammatory and cardiovascular activities of AS are mediated through inhibition of COX and LOX pathways. This new information provides a theoretical basis for development of new anti-inflammatory approaches targeted to COX and LOX enzyme activity. A number of metabolically active products are yielded by COX and LOX pathways of AA metabolism which, in various inflammatory conditions play critical roles (Saeed 2007b). Compounds with activities against both of these enzymatic pathways would be ideal for treating certain inflammatory conditions (Saeed, 2003b). The information produced by our study increases our understanding about these compounds and their roles 
for targeting COX and LOX pathways for development of new anti-inflammatory approaches. Since no effective inhibitor is currently available to block the COX and LOX pathways, butanolic fraction of AS might be valuable for the isolation of pure compounds that show dual inhibition of COX and LOX pathways.

\section{REFERENCES}

- Ahmad, N., Shinwari, Z.K., Hussain, J., Ahmad, S., Abbas, G., Zada, M., Ahmed, N., Iqba,1 J. (2011). Antiglycation, platelet aggregation, antioxidant, cytotoxicity and phytotoxic activities of the crude extract/fractions of Rhynchosiareniformis. Journal of Pharmacy Research, 4 (10): 3721-3724.

- Ahmad, N., Shinwari, Z.K., Hussain, J., Ahmad, S., Abbas, G., Zada, M., Ahmed, N., Iqba,l J. (2011). Biological Evaluation of the Crude Extracts/Fractions of Nepetaleavigata and Nepetakurramensis. Journal of Pharmacy Research, 4 (10): 3472 3474.

- Ahmed, S., Gul, S., Gul, H., Bangash, M.H. (2013). Dual Inhibitory Activities of AdhatodaVasica against Cyclooxygenase and Lipoxygenase. International Journal of Endorsing Health Science Research, 1 (1), 14-17.

- Aslam, R., Saeed, S.A., Ahmed, S., Connor, J.D. (2008). Plasma lipoproteins inhibit platelet aggregation and arachidonic acid metabolism in experimental hypercholesterolemia. Clinical and Experimental Physiology and Pharmacology, 35(5-6):656-62.

- Bukhari, I.A., Khan, R.A., Gilani, A.H., Ahmed, S., Saeed, S.A. (2010). Analgesic, anti-inflammatory and anti-platelet activities of the methanolic extract of Acacia modesta leaves. Inflammopharmacology, 18(4):187-96.

- Butt, M.S., Tahir-Nadeem, M., Khan, M.K., Shabir, R., Butt, M.S. (2008). Oat: unique among the cereals. Eur J Nutr, 47(2):68-79.

- Dimberg, L.H.,Gissén, C., Nilsson, J. (2005). Phenolic compounds in oat grains (AS L.) grown in conventional and organic systems. Ambio, 34(4-5):331-7.

- Feng, B., Ma, L., Yao, J., Fang, Y., Mei, Y., Wei, S. (2013). Protective effect of oat bran extracts on human dermal fibroblast injury induced by hydrogen peroxide. J Zhejiang UnivSci B, 14(2): 97-105.

- Gul, S., Ahmed, S., Gul, H., Kaneez, K.F. (2010). Investigating the Protective Effect of Solanummelongena. Asian Journal of Health, 1(1):4-9243.

- Hussain, J., Khan, F.U., Gilani, S.A., Abbas, G., Ahmed, S. Khan, A.U., \&Choudhary, M.I. (2010). Antiglycation, antiplatelets aggregation, cytotoxic and phytotoxic activities of Nepetasuavis'. Lain. American Journal of Pharmacy, 29 (4): $573-$ 8.

- Hussain, J., Jamila, N., Gilani, S.A., Abbas, G., and Ahmed, S (2009). Anti-Platelet aggregation, antiglycation, cytotoxic, phytotoxic and antimicrobial activities of extracts of Nepetajuncea. African Journal of Biotechnology, 8(6): 935-940.

- Imran, I., Hussain, L., Ahmed, S., Rasool, N., Rasool, S., Abbas, G., Ali, M.Y. (2012). Antiplatelet activity of methanolic extract of Acacia leucophloea bark. Journal of Medicinal Plants Research, 6 (25), 4185.

- Ji, L.L., Lay, D., Chung, E., Fu, Y., Peterson, D. M. (2003). Effects of avenanthramides on oxidant generation and antioxidant enzyme activity in exercised rats. Nutrition. Research, 23:1579-1590.

- Katz, D.L., Nawaz, H., Boukhalil, J., Chan,W., Ahmadi, R., Giannamore, V., Sarrel, P. M. (2001). Effects of oat and wheat cereals on endothelial responses. Prev. Med, 33:476-484.

- Liu, L., Zubik, L., Collins, F. W., Marko, M., Meydani, M. (2004). Theantiatherogenic potential of oat phenolic compounds. Atherosclerosis, 175:39-49.
- Masood, S.B., Muhammad, T.N., Muhammad, K.I.K., Rabia, S., Mehmood, S.B. (2008). Oat: unique among the cereals European. J. Nutrition, 47(2):68-79.

- Matsukawa, T., Isobe, T., Ishihara, A., Iwamura, H. (2000). Occurrence of avenanthramides and hydroxycinnamoylCoA:hydroxyanthranilate N-hydroxycinnamoyltransferase activity in oat seeds. Z. Naturforsch, [C], 55:30-36.

- Nie, L., Wise, M. L., Peterson, D. M., Meydani, M. (2006a). Avenanthramide, a polyphenol from oats, inhibits vascular smooth muscle cell proliferation and enhances nitric oxide production. Atherosclerosis, 186:260-266.

- Nie, L., Wise, M., Peterson, D., Meydani, M. (2006b). Mechanism by which avenanthramide-c, a polyphenol of oats, blocks cell cycle progression in vascular smooth muscle cells. Free Radic. Biol. Med, 41:702-708.

- Peterson, D.M. (2001). Oat antioxidants. J. Cereal. Sci., 33(2):115-129.

- Saeed, S.A., Khan, S.K., and Ahmed, S. (2003a). The Inhibition of Prostaglandin Biosynthesis by Human Plasma and its Relationship to Albumin and Haptoglobin. Journal of Biological Sciences, 3 (12): 1188-1202.

- Saeed, S.A., Ahmed, S., Ali, A. (2003b). A New Function of Human Haptoglobin: Endogenous inhibition of prostaglandin biosynthesis and its relation to hemoglobin binding. Journal of Medical Sciences, 3(5-6):344-357.

- Saeed, S.A., Rasheed, H., Ali, T.H., and Ahmed, S. (2004). Mechanisms of inhibitory actions of cyclooxygenase-2 inhibitors in human platelets. Journal of Biological Sciences, 4 (4): 515 520.

- Saeed, S.A., Motiwala, A., Qureshi, Z.U., Khan, R., Ali, A., Quadri J., and Ahmed, S. (2006). Mechanisms of platelet aggregation mediated by G-protein coupled receptors in human platelets. Journal of College of Physicians and Surgeons Pakistan, 16 (2): 167.

- Saeed, S.A., Connor, J.D., Quadri, J., Tasneem, S., Ahmed, S., Mesaik, M.A., Choudhary, M,I. (2007a). Inhibitors of phosphatidylinositide 3-kinase: effects on reactive oxygen species and platelet aggregation. Pharmacol Reports, 59: 238 243.

- Saeed, S.A., Ahmad, N., Ahmed, S. (2007b). Dual inhibition of cyclooxygenase and lipoxygenase by human haptoglobin: its polymorphism and relation to hemoglobin binding. Biochem. Biophys. Res. Commun, 353: 915 - 920.

- Saltzman, E., Das, S. K., Lichtenstein, A. H., Dallal, G. E., Corrales, A., Schaefer, E. J., Greenberg, A. S., Roberts, S. B. (2001). An oat-containing hypocaloric diet reduces systolic blood pressure and improves lipid profile beyond effects of weight loss in men and women. Journal of Nutrition, 131:1465-1470.

- Schellekens, C., Perrinjaquet-Moccetti, T., Wullschleger, C., and Heyne, A. (2009). An Extract from Wild Green Oat Improves Rat Behaviour. Phytotherapy Research, 23, 1371-1377.

- Truswell, A. S. (2002). Cereal grains and coronary heart disease. Eur. J. Clin. Nutr, 56:1-14; 2002.

- Wichtl, M. (2002). Teedrogen und Phytopharmaka, 4. Auflage. WissenschaftlicheVerlags-Gesellschaft: Stuttgart, 72-74.

- Zia-Ul-Haq, M., Shahid, S.A., Ahmed, S., Ahmad, S., Qayum, M., and Khan, I.U. (2012a). Anti-platelet activity of methanolic extract of Grewiaasiatica L. leaves and Terminallachebula Retz. Fruits. Journal of Medicinal Plants Research, 6(10), 2029-2032. ᄀ - Zia-ul-Haq, M., Khan, B.A., Landa, P., Kutil, Z., Ahmed, S., Qayum, M., Ahmad, S. (2012 b). Platelet aggregation and antiinflammatory effects of garden pea, Desi chickpea and Kabuli chickpea. ActaPoloniaePharmaceutica, 69(4):707-11.

- Zia-Ul-Haq, M., Ahmed, S., izwani, G.H., Qayum, M., Ahmad, S., Hanif, M. (2012 c). Platelet aggregation inhibition activity of selected legumes of Pakistan. Pakistan Journal of Pharmaceutical Sciences, 25(4):863-5. 